\title{
Methodological insights: fuzzy sets in medicine
}

\section{$P$ Vineis}

Correspondence to: Paolo Vineis, Imperial College London, Department of Epidemiology and Public Health, Norfolk Place, London W2 1PG, UK; p.vineis@imperial.ac.uk

Accepted 3 July 2007

\section{ABSTRACT}

In this paper I wish to introduce some ideas about scientific reasoning that have reached the epidemiological community only marginally. They have to do with how we classify things (diseases), and how we formulate hypotheses (causes). According to a simplified and currently untenable model, we come to defining what a disease, or a protone or a chromosome, is by progressive simplification - that is, by extracting an essence from the individual characters of disease. At the end of this inductive process a single element, which guarantees the unequivocal inclusion in the category, is identified. This is what has been called "Merkmal-definition" (Merkmal meaning distinctive sign) - that is, the definition of disease would be allowed by the isolation of a crucial property, a necessary and sufficient condition, which makes that disease unique (and a chair out of a chair, a proton out of a proton, etc). However many objections have been raised by Wittgenstein, Eleanor Rosch and others to this idea: a Merkmal is not always identifiable, and more often a word is used to indicate not a homogeneous and unequivocal set of observations, but a confused constellation with blurred borders. This constellation has been called a fuzzy set and is at the basis of the semantic theory of metaphors proposed by MacCormac and the prototype theory proposed by Rosch. In this way the concept of disease, for example, abandons monothetic definitions, amenable to a necessary and sufficient characteristic, to become "polythetic." I explain how these concepts can help medicine and epidemiology to clarify some open issues in the definition of disease and the identification of causes, through examples taken from oncology, psychiatry, cardiology and infectious diseases. The definition of a malignant tumour, for example, seems to correspond to the concept of "family resemblance," since there is no single criterion that allows us to define unequivocally the concept of cancer: not morphology (there are borderline situations between benign and malignant), not clinical features, not biochemical or molecular lesions. In the case of schizophrenia, the problem of indetermination, as it has been defined, is even stronger. Mental disease probably cannot be distinguished from health in a clearcut way (according to a minimum set of necessary criteria), but it would have a fuzzy border with mental conditions that characterise normal subjects, through intermediate linking conditions.

The purpose of this paper is to tackle some epistemological problems encountered in medicine, in particular how diseases are defined and classified. A simplistic view, based on a linear advancement of biomedicine and on simply descriptive propositions, which become closer and closer to truth thanks to an increasing level of detail, is in fact no longer tenable.

This paper largely refers to Wittgenstein's philosophy, but also to the prototype theory as expressed by Eleanor Rosch. ${ }^{1}$ A clear and intriguing account of similar problems is contained in the book Sorting things out by Bowker and Star. ${ }^{2}$

\section{WHY IS THIS RELEVANT TO EPIDEMIOLOGY?}

In some way every biomedical researcher is searching for a kind of ultimate explanation of disease. So, we have been told that all cancers were due to mutations in p53 (some years ago), then it was the turn of epigenetics, and so on. It seems that looking for a single and simple explanation of things-and an ensuing unambiguous classification -is inherent in human psychology. However, we should also be aware that at least sometimes it is not possible to find such an explanation and such a classification. For example, people have struggled for years but still an unambiguous classification of viruses is not available. ${ }^{2}$ When we adopt a classification of disease (for example, International Classification of Diseases, tenth revision, ICD-10), or when we refer to the diagnostic criteria for disease, or when we think of causes, we usually imply that such activities are based on unequivocal criteria that sharply distinguish one disease from another one, or that relate a cause to its effect. This is very clear in the paradigm of "necessary and sufficient" causes. However, reality is very far from such interpretation. This paper tries to show why.

\section{A THEORY OF METAPHORICAL KNOWLEDGE}

The Austrian philosopher Wittgenstein put forward in his life two largely different-and to a certain extent opposite-philosophical theories of language and of truth. In his first conception of language Wittgenstein suggested an interpretation of the proposition as a "measurement tool," since he intended to show, in his first philosophical phase, that the only true knowledge is scientific knowledge (consisting in the description of "states of affairs"). He proposed that such knowledge was based on models, like in the reconstruction of a car accident in the court, which would be a faithful reproduction of the events described. The relation between proposition and state of affairs would be allowed by an unequivocal correspondence between words and objects (experience data), so that there is, between the components of the proposition, the same structural relation that exists among single elements of the "state of affairs"for example, names and verbs are in the same relation as cars, people and actions in the court reconstruction of the car accident. ${ }^{3}$

A descriptive proposition can also be compared to a miniaturised model of a ship, which has, on a lower scale, the same parts of the original. A model thus conceived has something that corresponds to the original and something that does not 
correspond, and the latter obviously is something inessential for the identification of the real object. But if all propositions were of this kind, there would be no progression in knowledge: advancement would consist just in increasing details - that is, the accuracy with which the original can be found in the model. This would not be a satisfactory account of how changes in knowledge paradigms occur; a situation in which the "original" (the true ship) is not in front of us, but is hidden, like disease, so that we have to explore it with repeated attempts. Let us consider scientific theories of cancer. In fact we never have "cancer" in its "essence" in front of us, but only different ways of looking at malignant tumours: a morphological modality, based on microscopic description of cancer tissues; a genetic modality based on alterations observed in DNA; a biochemical modality based on metabolic malfunction of cells, and so on. An even superficial history of theories of carcinogenesis can easily persuade us that there was not at all an increasing ability to describe details of "cancer" - that is, something fixed, existing "there" outside our paradigms. Therefore the use of a proposition as a "measurement tool" is a condition (perhaps) necessary but certainly not sufficient to describe knowledge processes. The figure of speech for which it is possible to think that changes in the course of time play a role, is a metaphor, which allows an enrichment of knowledge and not only an increase of detail.

In the theory of metaphor people have distinguished between those metaphors whose primary function is descriptive, and those whose function is suggestive. The former, called epiphors, are based on analogies in which the relation between the model and the "state of affairs" is obvious: "what corresponds" is thus prevailing in them. In the latter instead there is an anomaly, a lack of correspondence: they (diaphor) suggest new meanings by emphasising the dissimilarities among referents rather than their similarities. An epiphor is a proposition whose correspondence with reality is not discussed, while a diaphor can contain those surprising elements that are typical of literary communication. In the case of scientific theories, it is hypothesised that they are born as diaphors, and, if successfully validated, slowly become epiphors. For example, the word "force" as suggested by newtonian physics was subject to a metaphoric use, obtained by juxtaposition of the meaning of force typical of aristotelian mechanics and the new theory elaborated by Newton on action from a distance (gravity). The following experimental verification of the uses of this word transformed the diaphor into an epiphor, the expression of an accepted analogy instead of an uncertain and innovative suggestion. An example of a diaphor that is still alive, since there is no proof available of its existence, is the "tachyon" - that is, a particle able to travel at a speed greater than that of light (until now, by definition, no particle was considered to be able to travel at a speed more than light).

Metaphor, as a model of proposition that includes the two extreme types of diaphor and epiphor, can serve the purpose of representing knowledge in a dynamic way. In the case of cancer theories, the idea that cancer was an infectious disease, transmissible by personal contact was for a certain period a diaphor, then falsified by epidemiological research (most cancers do not transmit by contact, although cervical cancer is due to repeated, long-term exposure to human papillomavirus). The hypothesis that cancer is a genetic disease (in a broad sensethat is, a disease related to DNA alteration) is a still vital diaphor. And the hypothesis that it is an environmental disease is an epiphor supported by clear and numerous observations (for example, the fact that migrants acquire the risk of cancer of the population into which they migrate).
Philosophers such as MacCormac ${ }^{4}$ have proposed a theory of truth based on the success or failure of those that are called "root-metaphors," those which are constitutive of a certain theory-that is, were born as diaphors and served for the generation of relevant and crucial observations. It is in their use that the origin of scientific creativity has to be searched. One can think, for example, of the great divide between the newtonian conception [the world as a machine] and the leibnizian conception [the world as an organism] in terms of two radically different root-metaphors, in which the first turned out to be the winner, progressively becoming an epiphor, a kind of truth accepted without attrition.

\section{IS ALL KNOWLEDGE METAPHORICAL?}

From the preceding arguments one could draw the wrong conclusion that all knowledge is metaphorical and there is no direct and verifiable knowledge of the world. In other words, if all knowledge is metaphorical, what is the place, if any, of literal propositions? This problem was raised explicitly by some linguists and anthropologists: the "Sapir and Whorf thesis" claims in fact that the perception of the world is influenced, or even determined, by the linguistic structures of which we make use. On the basis of his observations of Hopi Indians, Whorf noticed that their conception of time, of causality and of movement was greatly conditioned by the availability of linguistic structures quite different from the Western ones. ${ }^{5}$ The Sapir-Whorf thesis, however, has been falsified by anthropological observations suggesting that for some basic aspects of human perception-for example, colours, there is an extraordinary linguistic similarity among different cultures. In particular, it has been observed that the borders that distinguish chromatic categories are constantly set in the same points of the spectrum. It seems, in other words, that for the human primary functions, the material interaction with the world, humans have a simple and reproducible linguistic structure, not conditioned by the culture to which they belong. A similar analysis has been made by Hart and Honoré ${ }^{6}$ when talking about the concept of "cause," whose primitive idea would date back to the simplest material interactions with the world, while the more sophisticated ones would be derived by analogical extension. This simpler use of language is what is usually defined as literal-that is, the description of prototypal situations like the scale of colours or the "efficient cause" of Aristotle.

What happens, however, with simple objects like a chair, that do not correspond to prototypal universal situations?

\section{THE UNDERSTANDING OF WORDS: CHAIR, DISEASE}

Let us see how, according to the analysis done by Ludwig Wittgenstein ${ }^{7}$ and, more recently, by Rosch, ${ }^{1}$ the use of the word "chair" and its understanding occur in practice. If I show a chair and pronounce its name, a listener who does not understand my language could think I refer to the wood it is made of, the legs, its red colour, the shallow surface or whatever other property. It is not just by the direct act of showing a prototype that the use of language is learnt, both because in showing the prototype I can be misunderstood about the meaning of my gesture (do I refer to the material, the colour or a part of the chair?), and because the listener will find herself in front of many other chairs whose correspondence with the prototype is only partial. It is not memorising an abstract model that allows her to link the word chair with the relevant objects, but instead a continuous work of adaptation and rearrangement 
of the concrete uses of words that the community of speakers makes.

The use of the word is strictly connected, in this work of understanding, with the use of the designated objects. The linguistic interactions are also a material interaction with the world. Even if not all chairs have four legs, like the prototype, even if they do not have arms, and so on, the subject will identify, when examining the forms and functions of various chairs, those characters that allow her to talk about chairs in an appropriate way. Therefore, in addition to the description of prototypal situations, the literal use of language is also the product of a material interaction with the world. MacCormac has defined the literal as the use of ordinary language to express concrete objects and events, noting that, however, the borders between literal and metaphorical are not clear cut. It is likely that, beyond prototypal situations, most literal uses of the language are "old epiphors" whose ambiguity has been progressively lost in the course of centuries. It is very suggestive, in any case, that the development and use of language are, in this interpretation, linked with a strict interaction with the material world: according to the concept used by both Wittgenstein and Simone Weil, "originally was action."

The idea, originally suggested by Wittgenstein and then elaborated by MacCormac and Rosch, that all objects corresponding to the word chair are not a fixed prototype-recalled by our mind in front of concrete chairs-is crucial to understand how the model of abstraction of an essence from observable entities can be abandoned. According to this simplistic inductive model, we come to defining what a chair is-or a protone or a chromosome-by progressive simplification, by giving up with the individual characters of single chairs; at the end of this inductive process a nuclear element, a single element that guarantees the unequivocal inclusion in the category, would be identified. This is what philosophers call "Merkmal-definition" (Merkmal meaning distinctive sign) - that is, the definition would be allowed by the isolation of that unique crucial property-a necessary and sufficient condition - that makes a chair out of a chair, a proton out of a proton, etc. However many objections have been raised by Wittgenstein to this idea: a Merkmal is not always identifiable, and more often the word is used to indicate not a homogeneous and unequivocal set of

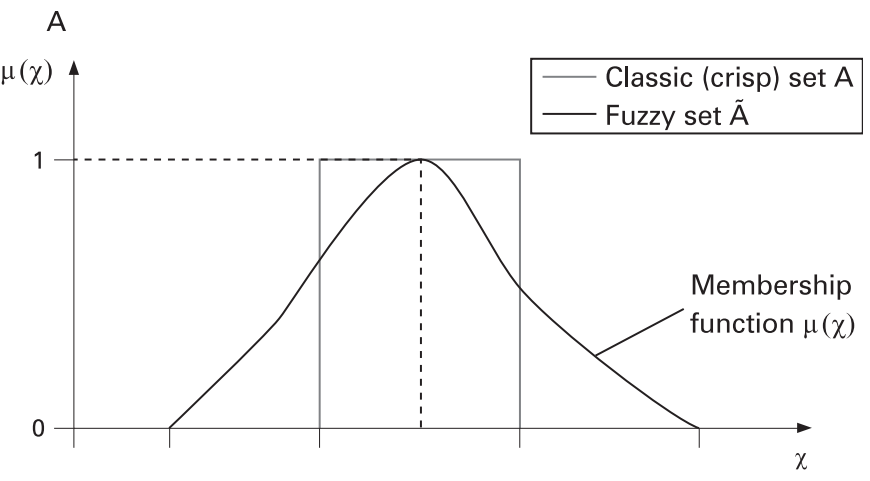

B

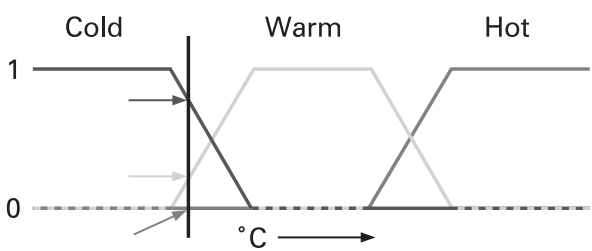

Figure 1 Fuzzy sets (from Wikipedia). observations, but a confused constellation with blurred borders. ${ }^{8}$ This constellation has been called a fuzzy set, and is at the basis of the semantic theory of metaphors by MacCormac. Within the fuzzy set called "chair" we have, in a central position, the definition of chair that one can find in the dictionary; around this prototypal meaning there is a cloud of meanings, which on the one hand include ambiguous objects that serve to seat but do not have the shape of chairs, and at the other extreme ambiguous objects that look like chairs but do not serve for seating. The concept of chair gives up with a monothetic definition that is being amenable to a necessary and sufficient characteristic, to become "polythetic."

\section{FUZZY SETS}

A fuzzy set is a set in which membership is not crisp (yes or no), but comes in degrees according to a function, as figure 1 shows (part A). Part B shows that there is not a crisp distinction between cold and hot, but an intermediate category of warm with fuzzy borders. Different states have ambiguous memberships-that is, can belong in different categories (have a function of membership in different categories).

The fact that many words are used as fuzzy sets-that is, with flexible meanings with blurred borders, assures the possibility of change and progress in knowledge. This is particularly true if the word plays an important role in a metaphorical proposition. If the word had a fixed meaningthat can be deciphered through a dictionary-the metaphorical extension that allows the development of new metaphors would not be possible. Diaphors would not exist if a word had not a prototypal meaning plus other meanings showing weaker links with the prototype within the fuzzy set. Inclusion in the category of objects defined by the word is determined by the "distance of similarity with the prototype"-that is, by similarities and dissimilarities between the object and the prototype. Strictly speaking, in Wittgenstein's conception there is not necessarily a prototype, but a sequence of overlapping correspondences; the comparison that has been proposed is with a rope made of many different threads, none of which however is long like the rope itself: thus, in the case of the chair we have at the centre the prototypal chair, on one side a sequence of objects without four legs but serving to seat, and on the other side a sequence of objects that have four legs but serve many different purposes.

Metaphor comes from the juxtaposition of words apparently in conflict, at least in a diaphor; this contrast is sensible only if the meaning of the two words can be "stretched" sufficiently so as to allow the identification of a perspicuous image of the world. The fact that I say that cancer is formed by "crazy cells" has some possibility of succeeding only if the concept of craziness can be expanded in order to cover the biological behaviours of those cells that, invading surrounding tissues, conferred to breast cancer the aspect of a crab (= cancer) (when the image of "cancer" was coined). MacCormac invites us to imagine the semantic space of a metaphor as a space in which different words are linked to each other by vectors. The length of each vector determines how strictly the two words are related. For example, "animated" will have a short vector that links it with "man" and "dog," but a very long vector that links it to "locomotive." Every language creation, from the poetic ones to the ethical and the scientific ones, is based on the proposal of new vectors that link, in a more or less convincing way, different words. There is obviously a relation between the length of vectors and belonging to a fuzzy set: an object with an ambiguous status, say between a vehicle and a children's game 
(like a rudimentary chart) will have a weak linkage with both fuzzy sets-that is, it will be far from the prototypes of both categories, and will tend to possess very long vectors that link it to other objects that could be reasonably linked with the prototypes. A long vector that links two objects indicates that it is unlikely that the two objects can be reasonably included in a same fuzzy set; but this cannot be easily established a priori: who would have thought of a connection between crabs and madness, as in cancer?

However, it is clear that not all diaphors are equally perspicuous in terms of knowledge. As Arthur Koestler said, "new ideas appear as mutations: most of them are useless, the same as jokes of nature whose fate is to perish." Koestler himself has compared the creative act to the release of an emotional energy in excess, suggesting a relation between knowledge and emotion.

If we accept that new knowledge is basically metaphorical, it does not occur thanks to simple correspondences or "isomorphisms" between propositions and states of affairs, as in Wittgenstein's Tractatus (first) conception. This conception was deeply affected by the Western tradition of the "mind's eye" - that is, knowledge as an act of seeing, according to which we know because there is a correspondence between the world's structure and the reconstruction we make of it in our mind. In this description of philosophy as the mirror of nature (in Richard Rorty's words $)^{9}$ the basic idea is that deepening our knowledge is the same as improving the details in comparison with the original, thanks to a work that is a progressive clarification (abstraction) of the concepts to which the language's words correspond.

How is the relation between metaphor and truth set, considering that those who formulate a new diaphor put themselves intentionally in the position of uttering an apparently false proposition? It is clear, first of all, that in this perspective it is necessary to abandon an absolute concept of truth, and introduce the idea of degrees of truth. Metaphor is a hypothetical utterance that needs verification. Truth or falseness are judged from how the different components of language link to each other: there is not a real direct comparison of constitutive parts of a metaphor with a state of affairs; rather, the words included in the basic similarity that underlies the metaphor are compared with events in the empirical world to establish the credibility of the similarity. As MacCormac claims: "A person who wants to formulate a new hypothesis or to express a deep emotion stretches the language metaphorically in order to produce new and deep meanings; certainly she does not create intentionally false propositions."

\section{DEGREES OF TRUTH}

There is considerable similarity between this conception of metaphor and the ideas that Wittgenstein expressed in the "Lecture on Ethics," - for example the tendency of humans to go beyond the cage of their language. Truth or falsity of a metaphor are clearly in association with the context of interpretation, since the metaphor tends to be false if taken literally and true if taken figuratively. To understand a metaphor implies that we have a stereoscopic vision that allows us to identify in the mean time the subject and the predicate, though they can be very far from each other.

Let us try for a moment to think of an application of the theory of metaphor to the field of ethics, instead of the field of knowledge. We could think, for example, that an apparently absolute principle like "do not kill" is in fact a fuzzy set, in which the categorical imperative is the central element having a prototypal nature, within a fuzzy set that can tolerate exceptions under special circumstances. In other words, the flexible nature of the fuzzy set allows us to face one of the most difficult problems of ethics, how to adapt principles to concrete circumstances. The fact that metaphor represents truth by degrees, instead of positing a dichotomous vision of what is good, allows us also to quantify in some way the degree of likelihood. Precisely, the degree to which similarities overcome dissimilarities is a measure of the metaphor being just a speculative possibility or expressing a probability. Beyond the actual likelihood of a quantification, giving up with a two-value logic (true/false) is however important because it carries some important consequences with it. The stiffness of some philosophers (Kant, but also the Wittgenstein Tractatus) in dealing with the dichotomy between scientific knowledge and ethical values was partly because of positing that the only sensible propositions were those whose reality is anchored to "yes" or "no." Metaphors do not refuse contradiction, as classical logic would like, since they can be true and false in the meantime. Truth and falsity, strictly speaking, become the limits of the continuum of truth that is expressed in a metaphor: precisely, absolutely true is a proposition that has entered into the ordinary language (a dead epiphor), while absolutely false is an empirical proposition that has been falsified or is inconsistent with the rest of our background knowledge (an abandoned diaphor).

\section{A COUPLE OF CAVEATS}

Problems are of course more complex than described here. Philosophers have much more to say on the subject. However, there are at least two aspects that need to be addressed briefly to avoid misunderstanding. Firstly, the fact that language works through metaphors and fuzzy sets does not mean that we cannot follow rules. As Wittgenstein showed, a rule cannot be always followed in a literal way. Sometimes a literal interpretation makes the rule simply ludicrous. For example, there is no rule that specifies, in the playing of tennis, how high the ball can and should be thrown. It is a matter of convenience and the ability of the player. The rule in this case is implicit in the general purpose of the play. This means that some rules of tennis are explicit and stiff-that is, are literal and cannot be violated, while others are fuzzy and depend on convenience.

Related to this there is the problem of relativism. To say that language is used in a fuzzy way and that there are degrees of truth does not mean that absolute truth does not exist or cannot be found. Not everything is a matter of opinion, or relies on interpretation or even regressio ad infinitum. For example, there is no doubt that Nazi concentration camps existed: this is a matter of fact that rests on a large amount of evidence and does not require any kind of metaphorical extension of language to be appreciated.

\section{EXAMPLES FROM MEDICAL RESEARCH}

As I have shown elsewhere ${ }^{11}$ the definition and classification of cancer do not seem to rest on unequivocal criteria. In the case of malignant tumours, their definition itself seems to correspond to the concept of "family resemblance," since there is no single criterion that allows us to define unequivocally the concept of cancer: not morphology (there are borderline situations between benign and malignant), not clinical features, not biochemical or molecular lesions. In the case of schizophrenia, the problem of indetermination, as it has been defined, is even stronger. According to some psychiatrists, such as Silvano Arieti, 
"schizophrenic thought is present in everyday life." For this reason Hunt, against the reductionist attempts, claims that the schizophrenic is not understandable on the basis of a positivist logic of quantification, empirical verifiability, logical order. (S)he can become understandable instead by analogy with mental states like panic or rage-that is, according to a mechanism of analogical extension of everyday experience shared by people in good mental health. We go back, therefore, to the concept of "family resemblance": mental illness would not be distinguished from health in a clearcut way (according to a minimum set of necessary criteria), but it would have a fuzzy border with mental conditions that characterise normal subjects, through intermediate linking conditions. Even better, mental illness is a fuzzy costellation that is contiguous, on the one hand, with physiological manifestations typical of everyday existence and, on the other hand, with real physicochemical changes such as those of fever hallucinations.

On the ground of the search for causes, the experience accumulated in the investigation of other chronic diseases allows us to set up a probabilistic and multifactorial model that, most likely, can also be tested in psychiatric pathology. The epidemiology of cancer and cardiovascular diseases has given up, for a long while, the aristotelian tradition based on necessary and sufficient causes, on the search for anatomically unequivocal lesions that would be typical of every disease-that is, with a simplified and abstract vision of causality. In the study of cancer the causal model distinguishes between external causes (exposures the individual has experienced in her life), the internal mechanism (a sequence of stages that can be documented at a molecular level) and the individual susceptibility based, for example, on a different ability to repair DNA. A similar multifactorial model, in which both external exposures (of a relational-environmental type) and a polygenic susceptibility are pertinent, can also be fruitful in psychiatry.

An additional difficulty comes from the fact that a large share of medicine, including psychiatry, is at the borderline between social sciences and natural sciences, and this situation makes it particularly vulnerable on methodological grounds. It is not granted, in fact, that the traditional tools are adequate to cover fields so disparate as the study of the consequences of exposure to chemical molecules and the study of psychosocial determinants of disease. In the meantime, in no other discipline as in the study of mental disease the advancement of knowledge has been characterised by the sequence of big "diaphors" rather than an increasing descriptive detail.

We can imagine a historical reconstruction of causality in medicine according to two vectors, the definition of the causal agent and the diagnostic process, and considering the transition from monothetic to polythetic concepts along both vectors. Smallpox is a monothetic disease both according to the definition vector (the poxvirus) and for symptoms and signs; tuberculosis is monothetic on the aetiological axis but polythetic on the symptoms/signs axis. And many "new" diseases (bulimia, psychiatric diseases, autoimmune disorders) are polythetic along both vectors. Some diseases have shifted from a monothetic form (with a "typical" presentation, characterised by evident symptoms, easily interpretable) to polythetic or borderline forms: one example is diabetes, which centuries ago was diagnosed by the sweet taste of urine and now is at the border of normality through an intermediate category (glucose intolerance). A very clear example of such a transition is told by Aronowitz, "from myalgic encephalitis to yuppie flue."12 In 1934 in California there was an apparent epidemic of poliomyelitis, with as many as 2648 cases reported in the first

\section{What is already known}

When we adopt a classification of disease (for example, ICD-10), or when we refer to the diagnostic criteria for disease, or when we think of causes, we usually imply that such activities are based on unequivocal criteria that sharply distinguish one disease from another one, or that relate a cause to its effect. This is very clear in the paradigm of "necessary and sufficient" causes. However, reality is very far from such interpretation. While we accept now that chronic diseases are multifactorial- that is, are due to "constellations" of risk factors, and while we accept, for example, that certain diseases like schizophrenia require a constellation of signs or symptoms to be diagnosed, still we are reluctant to accept that this is the rule and not the exception. Therefore, although it is already known that in special circumstances a "fuzzy" interpretation is needed, the idea of "fuzzy sets" has not been generalised as it should be.

\section{What this paper adds}

The present paper presents to epidemiologists, in non-technical terms, the idea that concepts like disease, cause, prognosis, etc, are "fuzzy sets" - that is, they are not amenable to a simple and unequivocal definition based on a single necessary characteristic. For example, the "cause" of lung cancer is not a single exposure or a single genetic trait, although we know that $90 \%$ of lung cancers are caused by tobacco. Or, mental disease cannot be distinguished from health in a clearcut way (according to a minimum set of necessary criteria), but it seems to have a blurred border with mental conditions that characterise normal subjects, through intermediate linking conditions. These issues have been treated in a technical way-for example, by Sadegh-Zadeh, Rosch and others, but here they are presented in an accessible way according to recent philosophical tradition.

seven months of the year. In fact, the symptoms were mild (it was not the classic paralytic form), to the point that several commentators spoke of "collective hysteria." Something very similar happened in the 1980s with the "chronic fatigue syndrome," a condition characterised by weakness and muscle pain, which arose mainly after a viral infection in middle-class subjects. Also in this case the lack of objective signs and, therefore, of a diagnostic test, and the lack of an aetiological explanation, make of the disease a typically polythetic condition, which includes probably both genuine post-viral syndromes and psychological distress, or even aspecific chronic tiredness (the yuppie disease).

Another case described by Aronowitz, in which a disease has been rediscovered after a long time, is Lyme disease. This disease is symptomatologically polythetic, being characterised, in a variable proportion, by serious joint pain, sore throat and dermatological manifestations. An epidemic of the disease occurred in Lyme, a small town in Connecticut, in the 1970s and-mainly after the pressure exerted by one of the victims, Polly Murray - in 1982 it was found that it was caused by a Rickettsia bacteria borne by ticks, called Borrelia burgdorferi. What is really interesting is that the Lyme disease was interpreted as an entirely new disease, while its description corresponds quite clearly to a condition already known in 
Europe since 1910 as "chronic migrating erythema." The European cases had a symptomatology that was mainly dermatological, and the transmission by ticks was already known. Lyme disease is, therefore, a clearly polythetic condition although aetiologically monothetic (transmitted by ticks, as was already known in 1910; and more precisely caused by Borrelia burgdorferi, as we have known since 1982).

A third example that is particularly relevant to the link between polythetic classifications, fuzzy sets and metaphor is coronary disease. Still according to Aronowitz, coronary disease is a cluster of attributions at different levels of reality (anatomical, physiological, symptomatological). It is a rhetorical short-cut, since the correspondence among attributes at the different levels is imperfect; the entity has a clear practical importance, but also generates misunderstanding about the material substrate for certain symptoms. More generally, the allegation-typical of mechanistic metaphors-that behind certain symptoms there are always anatomical alterations makes the pharmacological treatment more convincing and perhaps more effective, but represents only one component of truth.

\section{CONCLUSIONS}

Like in other scientific disciplines, a simplistic inductive theory is no longer tenable in medicine. A more complex model based on fuzzy sets and metaphors (in MacCormac's technical sense) ${ }^{4}$ is needed to describe the advancement in knowledge in different fields of medicine, encompassing the definition of diseases and the identification of causes. Things are more complicated, however, in medicine than in other domains, because medicine in fact is not exactly a natural science but is at the border between natural and social disciplines.

Acknowledgements: I am grateful to Miquel Porta, Ana Soto, Luis David Castiel and two anonymous reviewers for very thoughtful comments.

Competing interests: None.

\section{REFERENCES}

1. Rosch E, Lloyd B, eds. Cognition and categorization. Hillsdale, NJ: L Erlbaum Associates, 1978.

2. Bowker GC, Star SL. Sorting things out. Cambridge, MA: MIT Press, 1999

3. Wittgenstein L. Tractatus logico-philosophicus. London: Routledge, 2001.

4. MacCormac ER. A cognitive theory of metaphor. Cambridge, MA: MIT Press, 1988.

5. Whorf BL. Language, thought and reality: selected writings. Cambridge, MA: MIT Press, 1956.
6. Hart HL, Honoré AM. Causation in law. Oxford: Oxford University Press, 1985

7. Arrington RL, Glock HJ. Wittgenstein's philosophical investigations": text and context." London: Routledge, 1992.

8. Baker GP, Hacker PMS. Meaning and understanding. Chicago: University of Chicago Press, 1985.

9. Rorty R. Philosophy and the mirror of nature. Princeton: Princeton University Press, 1992.

10. Wittgenstein L. A lecture on ethics. The Philosophical Review 1965;74:3-12.

11. Vineis P. Definition and classification of cancer: monothetic or polythetic? Theor Med 1993;14:249-56.

12. Aronowitz RA. Making sense of illness: science, society and disease. Cambridge: Cambridge University Press, 1998.

\section{APPENDIX}

\section{Further reading}

A more technical account of the "fuzzy sets" theory in medicine can be found in the papers by Kazem Sadegh-Zadeh, for example:

- Sadegh-Zadeh K. Fundamentals of clinical methodology: 2. Etiology. Artificial Intelligence in Medicine 1998;12:227-70

- Sadegh-Zadeh K. Fundamentals of clinical methodology: 3. Nosology. Artificial Intelligence in Medicine 1999;17:87-108

- Sadegh-Zadeh K. Fuzzy health, illness, and disease. J Med Philosophy 2000;25:605-38

A very instructive book, with many examples of the "prototype" theory-as opposed to aristotelian criteria of classification-is Bowker GC, Star SL. Sorting things out. MIT Press, Cambridge MA: 1999.

A long philosophical debate on the scientific foundations of epidemiology has taken place in the recent past in epidemiological journals. Although the specific issue of fuzzy sets and metaphor has not been addressed, other issues such as the problem of induction, the logic of causal inference, the causal criteria, the problem of demarcation, the hypothesis-deductive method, the principle of falsifiability, scientific realism and the generalisability of the results of a sample to the population, have been discussed extensively. See for example:

- Weed D. Theory and practice in epidemiology. Ann NY Acad Sci 2001;954:52-62.

- Poole C. Induction does not exist in epidemiology, either. In: Rothman KJ, ed. Causal inference. Chestnut Hill, MA: ERI, 1988.

- Renton A. Epidemiology and causation: a realist view. J Epidemiol Community Health 1994:48:79-85

- Poole C. Commentary: Positivized epidemiology and the model of sufficient and component causes. Int J Epidemiol 2001;30:707-9

- Buck C. Popper's philosophy for epidemiologists. Int J Epidemiol 1975;4:159-68.

- Weed D. On the logic of causal inference. Am J Epidemiol 1986;123:965-79.

- Banegas JR, Rodriguez F, Del Rey Calero J. Popper and the problem of induction in epidemiology. Rev Esp Salud Publica 2000;74:327-39.

- Greenland S. Induction versus Popper: substance versus semantics. Int J Epidemiol 1998;27:543-8.

- Cole SR. Atheoretical science. A response to the poverty of Popperian epidemiology. Int J Epidemiol 1996;25:899-900.

- Karhausen L. The poverty of popperian epidemiology. Int J Epidemiol 1995;24:869-74.

- Maclure M. Inventing the AIDS virus hypothesis: an illustration of scientific vs unscientific induction. Epidemiology 1998;9:467-73. 